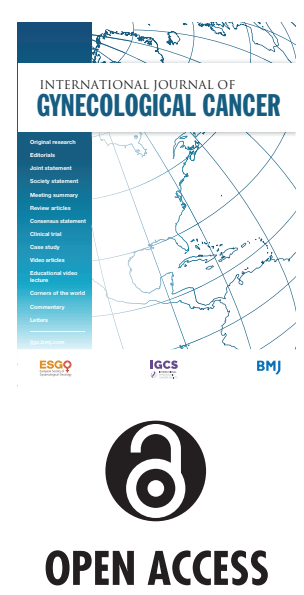

${ }^{1}$ Obstetrics and Gynecology, Shinshu University Graduate School of Medicine School of Medicine, Matsumoto, Nagano, Japan

${ }^{2}$ Radiology, Shinshu University Graduate School of Medicine School of Medicine, Matsumoto, Nagano, Japan

${ }^{3}$ Laboratory Medicine, Shinshu University Graduate School of Medicine School of Medicine, Matsumoto, Nagano, Japan

Correspondence to Dr Tsutomu Miyamoto, Obstetrics and Gynecology, Shinshu University Graduate School of Medicine School of Medicine, Matsumoto 3908621, Nagano, Japan; tmiya@ shinshu-u.ac.jp

Received 12 May 2020 Revised 7 August 2020 Accepted 11 August 2020 Published Online First 3 September 2020

Check for updates

(C) IGCS and ESGO 2020. Re-use permitted under CC BY-NC. No commercial re-use. Published by BMJ.

To cite: Kobara H, Miyamoto T, Ando $\mathrm{H}$, et al. Int J Gynecol

Cancer 2020;30:1480-1487.

\title{
Limited frequency of malignant change in lobular endocervical glandular hyperplasia
}

\author{
Hisanori Kobara, ${ }^{1}$ Tsutomu Miyamoto (iD , ${ }^{1}$ Hirofumi Ando, ${ }^{1}$ Ryoichi Asaka, ${ }^{1}$ Akiko Takatsu, \\ Ayumi Ohya, ${ }^{2}$ Shiho Asaka, ${ }^{3}$ Tanri Shiozawa $^{1}$

\section{HIGHLIGHTS} \\ - Clinical diagnosis of lobular endocervical glandular hyperplasia was consistent with pathological diagnosis. \\ - The rate of malignant change of lobular endocervical glandular hyperplasia during follow-up was limited (1 2\%). \\ - Worsening cytology and increasing lesion size may be important signs of malignant change of lobular endocervical \\ glandular hyperplasia.
}

\section{ABSTRACT}

Introduction Although lobular endocervical glandular hyperplasia is a benign disorder of the uterine cervix, its potential as a precursor of minimal deviation adenocarcinoma has been reported. However, the natural history of the disease and the frequency of malignant change are not fully understood. We evaluated the frequency of malignant change of clinical lobular endocervical glandular hyperplasia and explored useful parameters indicating malignant change.

Methods The clinical courses of 175 patients with cervical multi-cystic lesions who visited Shinshu University Hospital between June 1995 and June 2019 were retrospectively analyzed. We examined the results of follow-up and outcomes of the patients diagnosed with lobular endocervical glandular hyperplasia and investigated the frequency of malignant transformation. Results Of the 175 patients, 15,84 , and 76 were clinically diagnosed with suspected malignancy, suspected lobular endocervical glandular hyperplasia, and suspected nabothian cyst, respectively. Of these patients, 69 patients with suspected lobular endocervical glandular hyperplasia were followed, and 12 underwent hysterectomy after a mean follow-up of 57.1 (range: 3-154) months due to lesion enlargement (increase in tumor diameter of $>20 \%$ ) and/or worsening cytology. Of these 12 patients, two had lobular endocervical glandular hyperplasia with atypia and one had minimal deviation adenocarcinoma. Of 69 patients, the rate of malignant change was 1.4\% (1/69). The growth rates of the lesions for these three patients during follow-up were significantly higher than those of nine patients who underwent surgery with lobular endocervical glandular hyperplasia without atypia and 48 follow-up cases of suspected lobular endocervical glandular hyperplasia. The cut-off value of the growth rate suggesting malignant transformation was $38.1 \%$ (84.6\% sensitivity and $100 \%$ specificity). Tumor size and cytology did not change in the remaining 57 cases continuing follow-up.

Conclusion An increase in tumor size and worsening cytology are important parameters for detecting malignant transformation of lobular endocervical glandular hyperplasia during follow-up. However, the frequency of malignant change of this disease may be limited. These results suggest that conservative management may be an option for clinical lobular endocervical glandular hyperplasia.

\section{INTRODUCTION}

Lobular endocervical glandular hyperplasia is morphologically characterized by multiple cysts of the uterine cervix, and was originally reported as a benign, proliferative disorder. ${ }^{12}$ However, subsequent studies suggested that such lesions could be a potential precursor of minimal deviation adenocarcinoma, which is a well differentiated form of gastric-type mucinous carcinoma. $^{34}$ Therefore, appropriate preoperative diagnosis is important for adequate management. Although the differential diagnosis has been considered difficult, we proposed a diagnostic protocol for lobular endocervical glandular hyperplasia/minimal deviation adenocarcinoma using magnetic resonance imaging (MRI), cervical Papanicolaou (pap) cytology, and gastric-type mucin, ${ }^{5}$ and verified the usefulness of our protocol in a retrospective follow-up study. ${ }^{6}$ Recently, the number of patients diagnosed with clinical lobular endocervical glandular hyperplasia has markedly increased in Japan due to a growing recognition of this entity. However, the natural history of this lesion is still poorly understood, and little is known about the frequency of malignant change.

It is important to detect a malignant change of this lesion as early as possible in patients undergoing surveillance but useful clinical indicators of this malignant transformation have yet to be established. In the present study, we retrospectively analyzed the clinical courses of patients with cervical multi-cystic lesions, including cases of clinical lobular endocervical glandular hyperplasia managed in our hospital, to investigate the frequency and useful clinical parameters of malignant transformation of this disease.

\section{METHODS}

The study was approved by the ethics committee of Shinshu University School of Medicine (No 4451), and the requirement for written informed consent was waived by the committee. A total of 175 women with multi-cystic lesions detected by transvaginal 


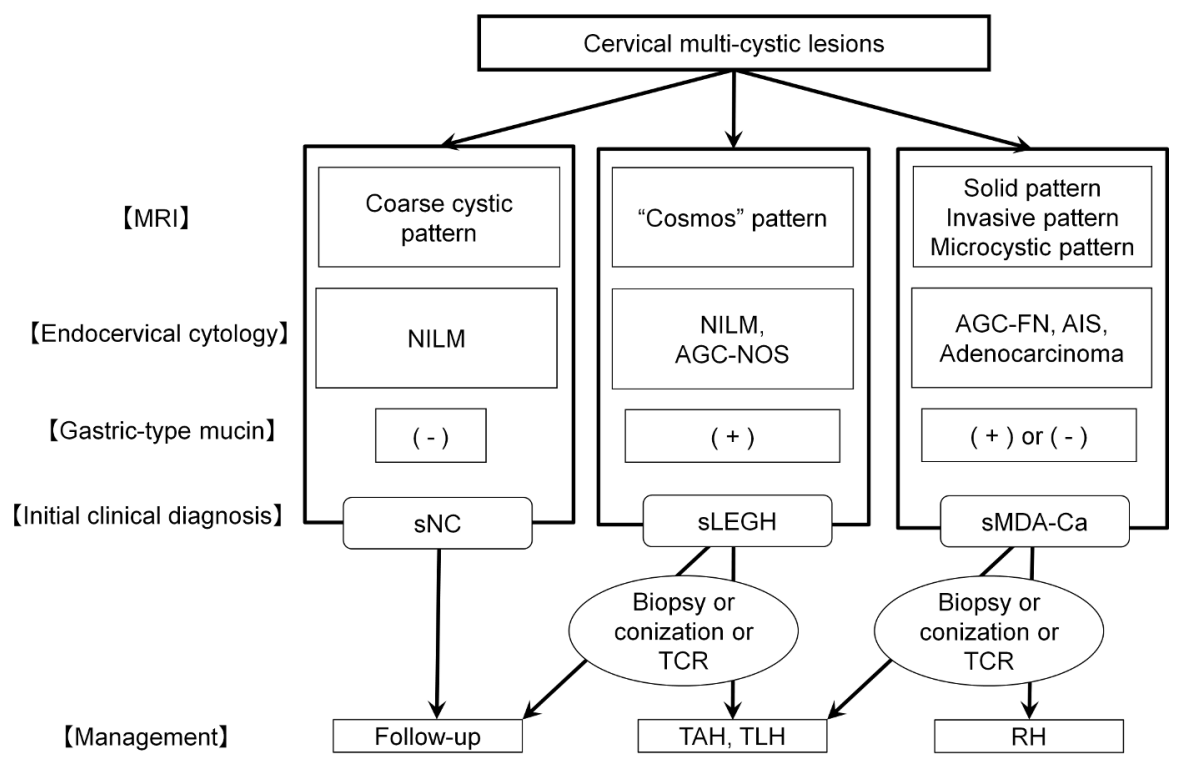

Figure 1 Diagnosis and management of cervical multi-cystic lesions. This is a modified version of Figure 1 in our previous report. ${ }^{6}$ AGC-FN, atypical glandular cells-favor neoplastic; AGC-NOS, atypical glandular cells-not otherwise specified; AIS, adenocarcinoma in situ; MRI, magnetic resonance imaging; NILM, negative for intraepithelial lesion or malignancy; RH, radical hysterectomy; sLEGH, suspected lobular endocervical glandular hyperplasia; sMDA-Ca, suspected minimal deviation adenocarcinoma or carcinoma; sNC, suspected nabothian cyst; TAH, total abdominal hysterectomy; TCR, transcervical resection; TLH, total laparoscopic hysterectomy.

ultrasonography, MRI, or computed tomography who visited Shinshu University Hospital between June 1995 and June 2019 were enrolled in the study. The study included 94 patients with a multicystic lesion, whose clinical courses were reported in our previous study. ${ }^{6}$ We retrospectively examined the results of follow-up and outcomes of the patients who were clinically diagnosed with lobular endocervical glandular hyperplasia and investigated the frequency of malignant transformation.

\section{Diagnosis, Treatment, and Follow-up}

In this study, 'cervical multi-cystic lesion' was tentatively defined by the presence of a cluster(s) of cysts in the cervix, and women having such findings were included in further analyses. In women with multiple cervical cysts, the primary clinical diagnosis and management were based on our protocol (Figure 1). ${ }^{56}$ In this protocol, the combination of three parameters (MRI, cervical pap cytology, and gastric-type mucin) was used for the clinical diagnosis, and we verified the usefulness of our protocol in a retrospective follow-up study. ${ }^{6}$ As we reported previously, T2 weighted MRI findings were classified as follows: (i) solid pattern (a solid component was noted, suggesting malignancy); (ii) invasion pattern (diffuse and solid high T2 weighted signal with an unclear margin in the cervical stroma, suggesting malignancy); (iii) cosmos pattern (diffuse or microcystic pattern surrounded by medium to large cysts, suggesting lobular endocervical glandular hyperplasia); (iv) microcystic pattern (aggregation of small cysts without peripheral large cysts, suggesting lobular endocervical glandular hyperplasia or malignancy); and (v) coarse cystic pattern (irregular aggregation of medium to large cysts without a solid or microcystic component, suggesting nabothian cysts).

A cytological diagnosis was made according to the Bethesda system. In particular, atypical glandular cells-not otherwise specified was regarded as suggesting lobular endocervical glandular hyperplasia without atypia, whereas atypical glandular cells-favor neoplastic suggested a lesion with atypia or carcinoma. Gastrictype mucin in the cervical mucus was examined using a latex agglutination assay (HIK1083 test, Kanto Kagaku, Tokyo, Japan) or by the detection of 'yellow' or 'orange' mucin in the cervical pap smear. ${ }^{7-9}$ Accordingly, the patient was clinically diagnosed with suspected malignancy when pelvic MRI revealed a predominant solid component or invasive lesion and/or atypical glandular cellsfavor neoplastic in the pap cytology. Patients with this category underwent immediate biopsy and hysterectomy. A patient was clinically diagnosed with suspected lobular endocervical glandular hyperplasia when a cosmos pattern on MRI, normal or atypical glandular cells-not otherwise specified cytology, and gastric mucin were noted. Patients with this category underwent surgery (conization or hysterectomy) or follow-up every 3-6 months. A patient was clinically diagnosed with suspected nabothian cysts when MRI showed coarse, multiple cysts with a clear margin, with negative cytology, and gastric-type mucin. The patients were recommended to undergo follow-up every 3-12 months. ${ }^{56}$

In this study, lesion size was measured by MRI in three dimensions. The dimension that showed the largest change was subjected to measurement as a growth rate. The growth rate (\%) was calculated according to the following formula; (current size-basal size)/ basal size $\times 100$. The current size indicated the diameter at the time of measurement during follow-up, and the basal size was the initial size or the minimum size during follow-up. Similar to the revised response evaluation criteria in solid tumors, an increase or decrease in lesion size was defined as $>20 \%$ enlargement or $30 \%$ reduction in growth rate, respectively. A pathological diagnosis was made according to the classification of the World Health Organization for glandular tumors and precursors of the uterine cervix. ${ }^{2}$ In this study, we defined lobular endocervical glandular hyperplasia with atypia 


\section{Original research}

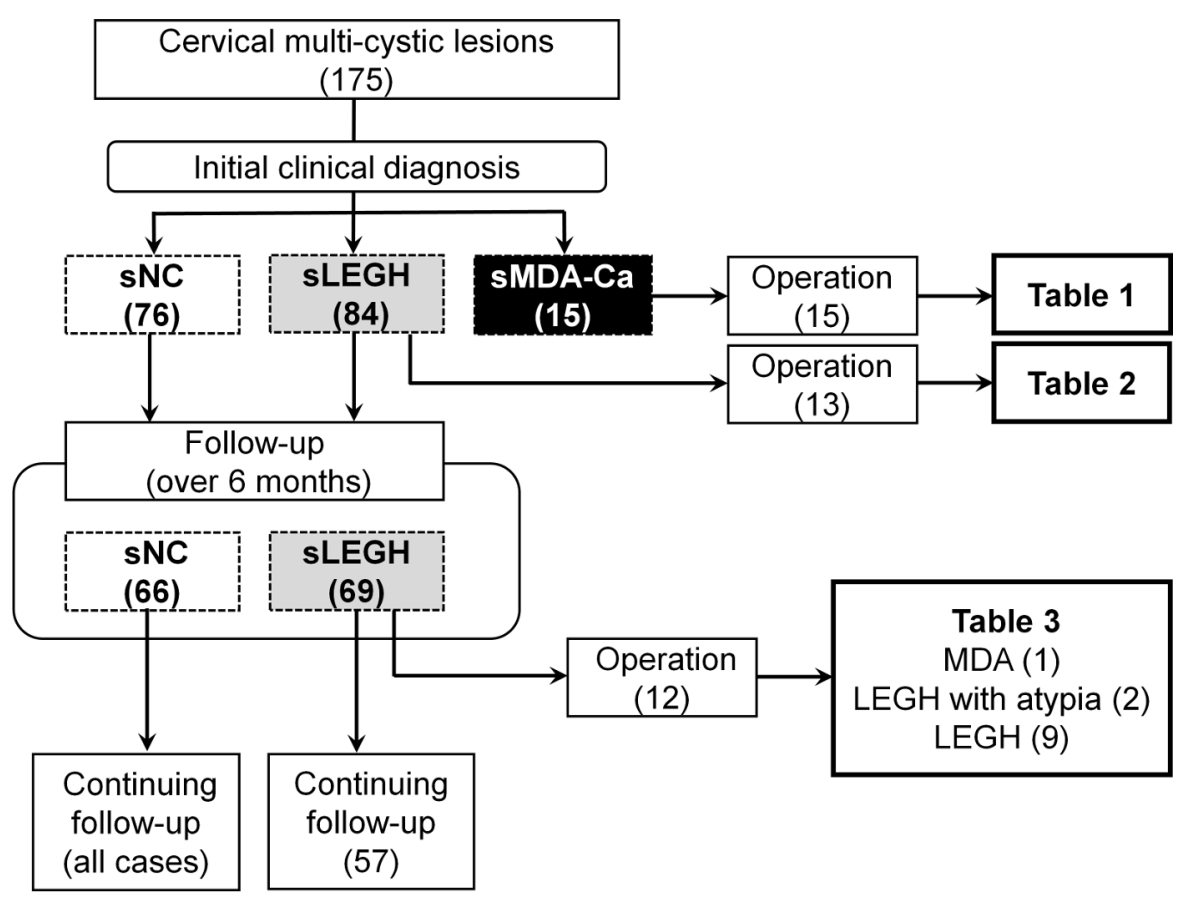

Figure 2 Summary of the clinical course of patients with multi-cystic lesions of the uterine cervix, managed according to our protocol. LEGH, lobular endocervical glandular hyperplasia; MDA, minimal deviation adenocarcinoma; sLEGH, suspected lobular endocervical glandular hyperplasia; sMDA-Ca, suspected minimal deviation adenocarcinoma or carcinoma; sNC, suspected nabothian cyst.

as follows: with at least one of the following morphologic features, including nuclear enlargement, irregular nuclear contour, distinct nucleoli, and/or loss of cell polarity.

The Mann-Whitney U test was used for statistical analysis. In patients undergoing follow-up, when MRI findings suggested signs of malignancy, such as an increase in lesion size, new detection of a solid or invasive pattern, lesions with a high signal intensity on diffusion weighted imaging, or when cellular atypia was detected by pap smear, follow-up was discontinued and surgery was recommended.

\section{RESULTS}

A total of 175 patients were included in the analysis and mean age was 46.5 years (range 24-76). The numbers of patients with suspected malignancy, suspected lobular endocervical glandular hyperplasia, and suspected nabothian cysts were 15,84 , and 76 , respectively. Mean ages ( \pm standard deviation) of patients in each group were $45.8 \pm 7.6,46.9 \pm 9.4$, and $46.0 \pm 8.9$; respectively. The proportions of patients complaining of watery discharge in each group were $26.7 \%, 52.4 \%$, and $21.1 \%$, respectively (Figure 2).

\section{Patients who Underwent Immediate Hysterectomy \\ Patients with Suspected Cancer}

The clinicopathological data of 15 patients in this category are summarized in Table 1. All patients underwent hysterectomy immediately after the clinical diagnosis (radical hysterectomy, $n=8$; total abdominal or laparoscopic hysterectomy, $n=7$ ) (Table 1). Of these 15 patients, 10 patients had adenocarcinoma (gastric-type mucinous carcinoma in nine and other in one). Malignant lesions were detected in nine patients by cervical biopsy. Eight showed a solid pattern on MRI, and 10 had an abnormal cytology.

Patients with Suspected Lobular Endocervical Glandular Hyperplasia

Eighty-four patients were initially diagnosed with this category (Figure 2). Of these 84 patients, 13 underwent hysterectomy immediately after the diagnosis (Table 2). Five of these 13 patients underwent a hysterectomy due to atypical cells detected by conization, the accidental association of cervical intraepithelial neoplasia grade 3, adenocarcinoma in situ, atypical polypoid adenomyoma, and endometrial carcinoma. The remainder selected hysterectomy for alleviation of symptoms or prophylaxis. The final histological diagnosis was lobular endocervical glandular hyperplasia in 11 patients (including with atypia in two, with cervical intraepithelial neoplasia III in one, and with adenocarcinoma in situ in one). The other two patients had tunnel cluster and nabothian cysts. The diagnostic accuracy for suspected malignancy and suspected lobular endocervical glandular hyperplasia was $66.7 \%$ (10/15) and $84.6 \%(11 / 13)$, respectively.

\section{Patients who Underwent Surveillance}

Patients with Suspected Lobular Endocervical Glandular Hyperplasia

Of 71 patients who did not select immediate hysterectomy, 14 and 5 underwent punch biopsy and conization, respectively. Eventually, 69 patients were followed up for more than 6 months (Figure 2, online supplementary Table 1), except for one (Table 3 ) who underwent hysterectomy 3 months after the initial diagnosis because of increasing lesion size. The clinical findings of all patients were observed every 3-12 months and the mean follow-up period was 
Table 1 Summary of 15 patients with suspected minimal deviation adenocarcinoma or carcinoma who underwent immediate hysterectomy

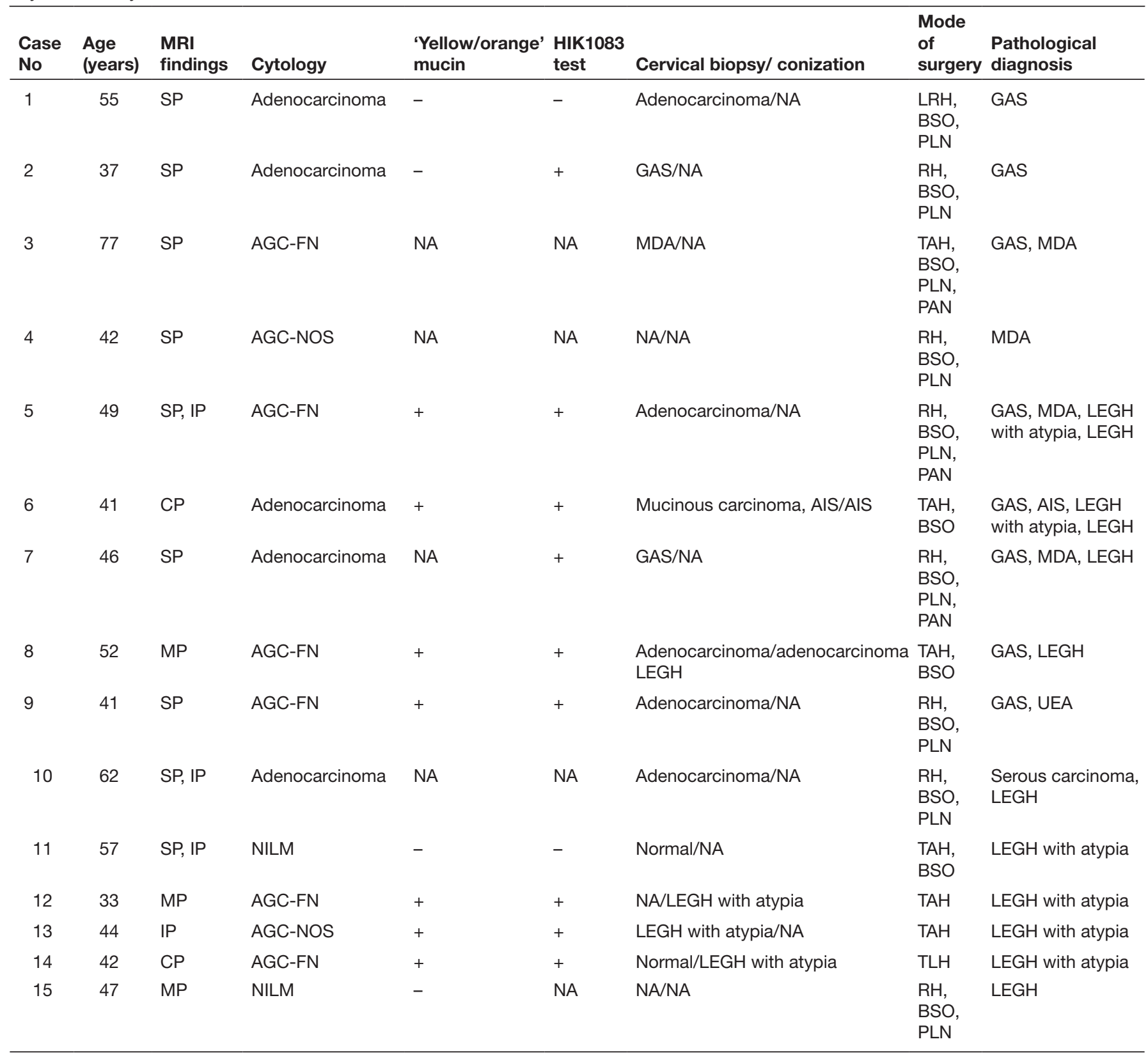

AGC-FN, atypical glandular cells-favor neoplastic; AGC-NOS, atypical glandular cells-not otherwise specified; AIS, adenocarcinoma in situ; BSO, bilateral salpingo-oophorectomy; CP, cosmos pattern; GAS, gastric-type mucinous carcinoma; IP, invasion pattern; LEGH, lobular endocervical glandular hyperplasia; LRH, laparoscopic radical hysterectomy; MDA, minimal deviation adenocarcinoma; MP, microcystic pattern; MRI, magnetic resonance imaging; NA, not available; NILM, negative for intraepithelial lesion or malignancy; PAN, para-aortic lymphadenectomy; PLN, pelvic lymphadenectomy; $\mathrm{RH}$, radical hysterectomy; SP, solid pattern; TAH, total abdominal hysterectomy; TLH, total laparoscopic hysterectomy; UEA, usual-type endocervical adenocarcinoma.

64.6 (range 3-154) months. Of the 69 patients in this category, 24 showed no change in lesion size on MRI, 19 showed an increase, and 14 showed a decrease. Of the 14 patients with a decreased lesion, $8(57.1 \%)$ had this size reduction after menopause.

Of the 69 patients under surveillance, 12 eventually underwent hysterectomy after follow-up for the reasons listed in Table 3. The mean duration of follow-up was 57.1 (range 3-154) months. Histologically, one had minimal deviation adenocarcinoma, two had lobular endocervical glandular hyperplasia with atypia, and the remainder had lobular endocervical glandular hyperplasia. It is noteworthy that the malignant change in one patient occurred after 5 years of follow-up..$^{10}$ Accordingly, the rate of minimal deviation adenocarcinoma or lobular endocervical glandular hyperplasia with atypia was $4.3 \%(3 / 69)$, and the rate of malignant change was $1.4 \%(1 / 69)$. Regarding the reasons for hysterectomy, 10 had suspected malignancy due to enlargement or deteriorating cytology and two were due to other diseases (atypical endometrial hyperplasia and adenomyosis) (Table 3). All 12 patients who underwent hysterectomy showed a cosmos pattern on MRI. Four patients also showed new findings suggestive of malignancy during follow-up 
Table 2 Summary of 13 patients with suspected lobular endocervical glandular hyperplasia who underwent immediate hysterectomy

\begin{tabular}{|c|c|c|c|c|c|c|c|c|}
\hline Case & Age & $\begin{array}{l}\text { MRI } \\
\text { findings }\end{array}$ & Cytology & $\begin{array}{l}\text { 'Yellow/orange' } \\
\text { mucin }\end{array}$ & $\begin{array}{l}\text { HIK1083 } \\
\text { test }\end{array}$ & $\begin{array}{l}\text { Cervical biopsy / } \\
\text { Conization }\end{array}$ & $\begin{array}{l}\text { Mode of } \\
\text { surgery }\end{array}$ & $\begin{array}{l}\text { Pathological } \\
\text { diagnosis }\end{array}$ \\
\hline 16 & 48 & $\mathrm{CP}$ & NILM & - & + & NA/NA & $\mathrm{TAH}$ & $\begin{array}{l}\text { LEGH with } \\
\text { atypia }\end{array}$ \\
\hline 17 & 33 & MP & AGC-NOS & + & + & $\begin{array}{l}\text { LEGH with atypia/ } \\
\text { LEGH with atypia }\end{array}$ & TLH & $\begin{array}{l}\text { LEGH with } \\
\text { atypia }\end{array}$ \\
\hline 18 & 40 & $\mathrm{CP}$ & NILM & - & + & LEGH/NA & TAH, BSO & LEGH \\
\hline 19 & 46 & $\mathrm{CP}$ & NILM & NA & + & Normal/NA & TAH & LEGH \\
\hline 20 & 57 & $\mathrm{CP}$ & AGC-NOS & + & + & NA/NA & $\mathrm{TAH}$ & LEGH \\
\hline 21 & 42 & CCP & AGC-NOS & - & + & NA/NA & $\mathrm{TAH}$ & LEGH \\
\hline 22 & 68 & $\mathrm{CCP}$ & NILM & + & + & NA/NA & TAH & LEGH \\
\hline 23 & 47 & $\mathrm{CCP}$ & NILM & - & + & Normal/NA & TAH, BSO & LEGH \\
\hline 24 & 53 & $\mathrm{CP}$ & AGC-NOS & + & + & NA/LEGH & TLH, BSO & LEGH \\
\hline 25 & 49 & MP & AGC-NOS & + & + & CIN3, LEGH/NA & TAH & CIN3, LEGH \\
\hline 26 & 52 & $\mathrm{CP}$ & AGC-NOS & + & + & $\begin{array}{l}\text { AIS, LEGH/AIS, } \\
\text { LEGH }\end{array}$ & TLH & AIS, LEGH \\
\hline 27 & 53 & $\mathrm{CP}$ & NILM & - & - & Normal/NA & TLH, BSO & Tunnel cluster* \\
\hline 28 & 48 & MP & NILM & - & + & NA/NA & TAH, BSO & $\mathrm{NC} \dagger$ \\
\hline
\end{tabular}

*With atypical polypoid adenomyoma.

†With endometrial carcinoma.

AGC-NOS, atypical glandular cells-not otherwise specified; AIS, adenocarcinoma in situ; BSO, bilateral salpingo-oophorectomy; CCP, coarse cystic pattern; CIN, cervical intraepithelial neoplasia; CP, cosmos pattern; LEGH, lobular endocervical glandular hyperplasia; MP, microcystic pattern; MRI, magnetic resonance imaging; NA, not available; NC, nabothian cyst; NILM, negative for intraepithelial lesion or malignancy; sLEGH, suspected lobular endocervical glandular hyperplasia; TAH, total abdominal hysterectomy; TLH, total laparoscopic hysterectomy.

(invasive pattern in one, solid pattern in another, and diffusion restriction in two others, online supplementary Figure 1, Table 3). However, no malignant component was detected in all four cases. The three patients showed worsening cytology from atypical glandular cells-not otherwise specified to atypical glandular cells-favor neoplastic, and the histological diagnoses of three patients were minimal deviation adenocarcinoma in one (Table 3) and lobular endocervical glandular hyperplasia in two. Gastric-type mucin was positive in all follow-up patients.

The three patients who developed minimal deviation adenocarcinoma or lobular endocervical glandular hyperplasia with atypia showed an increased lesion size. The average lesion enlargement rate of these patients was $91.2 \% \pm 44.9 \%$ (range +38.1 to $+148 \%$ ), whereas that of those pathologically (nine cases) or clinically (48 cases) diagnosed with lobular endocervical glandular hyperplasia was $-1.8 \pm 41.0 \%$ (range -61.5 to $+148 \%),(p=0.01)$. Receiver operating characteristic analysis was performed to test the specificity and sensitivity of the two groups. The cut-off value of the growth rate was $38.1 \%$ ( $84.6 \%$ sensitivity and $100 \%$ specificity) and the area under the curve was 0.923 (95\% confidence interval $0.821)$.

Patients with Suspected Nabothian Cysts

Sixty-six patients with this category underwent regular follow-up (Figure 2, online supplementary Table 1). The mean follow-up period was 52.4 (range 7-151) months. Of the 66 patients, 8 patients showed a decrease in lesion size on MRI, 9 showed no change, and 17 showed an increase in size. MRI was not available in 32 patients. No cytologic change was noted in any of the patients in this category.

\section{DISCUSSION}

Gastric-type mucinous carcinoma of the cervix shows gastrictype differentiation, and minimal deviation adenocarcinoma is defined as its well differentiated variant. ${ }^{2}$ These carcinomas were reported to be associated with aggressive behavior, chemotherapy resistance, and poor prognosis. ${ }^{11}$ However, clinical symptoms and morphological findings of these carcinomas were similar to those of lobular endocervical glandular hyperplasia. In those, the number of patients undergoing follow-up is also sharply increasing, especially in young women. Therefore, precise and early detection of its malignant change is extremely important. To address this issue, we focused on two clinical parameters indicative of its malignant change: lesion enlargement and worsening cytology. Accordingly, we detected three suspected lobular endocervical glandular hyperplasia cases associated with atypical cells in early stages.

The results of this study demonstrated that the rate of malignant change of clinical lobular endocervical glandular hyperplasia was $1.4 \%$, which is lower than previously considered. To our knowledge, this is the first report on the frequency of its malignant change. We recently reported that there was no driver gene mutation in four cases of non-atypical lobular endocervical glandular hyperplasia, suggesting its metaplastic nature. ${ }^{12}$ This result of genetic analysis may be consistent with the present data. In comparison with our 


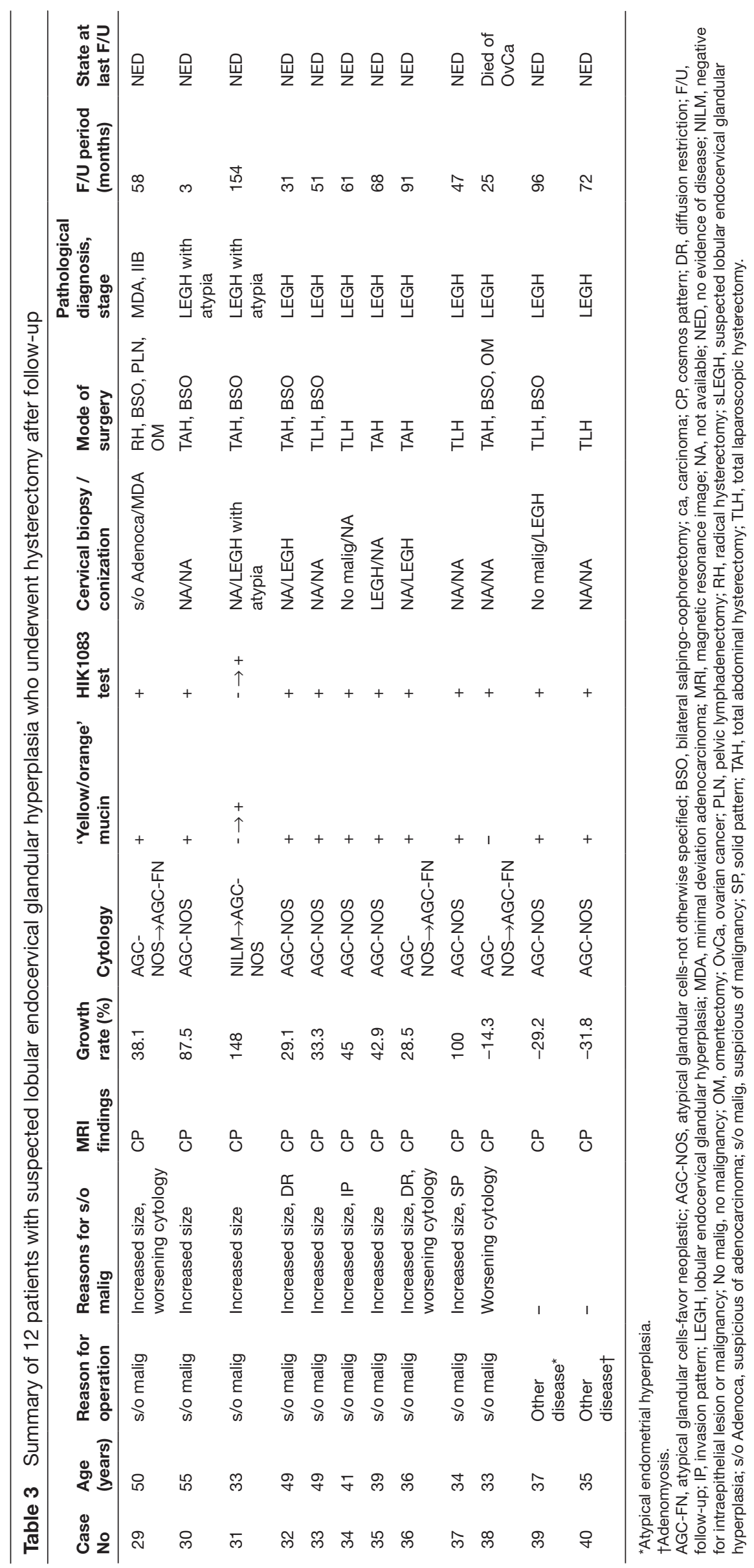




\section{Original research}

previous articles, ${ }^{56}$ the diagnostic accuracy of the present series was similar. In addition, a strength of our study was the identification of the malignant transformation ratio of suspected lobular endocervical glandular hyperplasia during follow-up (1.4\%, 1/69).

In this study, Iobular endocervical glandular hyperplasia with atypia was detected in two patients with suspected disease during follow-up (Table 3). Lesion enlargement was observed in both patients, but worsening cytology was not detected. Interestingly, one patient in this category was found to have minimal deviation adenocarcinoma (Table 3) and showed both lesion enlargement and worsening cytology (from atypical glandular cells-not otherwise specified to atypical glandular cells-favor neoplastic). ${ }^{10}$ This is consistent with a previous report. ${ }^{13}$ The present study suggests the importance of cytologic change rather than size change. However, it should be noted that two lobular endocervical glandular hyperplasia cases also showed worsening cytology, and some may be associated with an increase in size and worsening cytology, suggesting that the detection of worsening cytology in association with increased lesion size does not necessarily indicate the presence of malignancy.

Of 13 patients with suspected lobular endocervical glandular hyperplasia who underwent an immediate hysterectomy, four were associated with malignant/premalignant lesions (Table 2). The rate of association with malignant/premalignant lesions was high in this series $(30.1 \%, 4 / 13)$. However, the reason for immediate surgery in four patients was these malignant/premalignant lesions pathologically diagnosed before the operation instead of cervical multi-cystic lesions incidentally detected.

$\mathrm{MRI}$ is regarded as a useful tool in the diagnosis of multi-cystic lesions of the uterine cervix. ${ }^{56}$ In our study, we defined an increase in size $>20 \%$ as a 'size increase'. Although the number of patients with cancer was limited, the receiver operating characteristic analysis revealed that an increased lesion size of $38.1 \%$ or more may be closely associated with the onset of malignant transformation. There was a significant difference in the growth rate between typical lobular endocervical glandular hyperplasia and atypia or minimal deviation adenocarcinoma. Interestingly, this study revealed that $57.1 \%(8 / 14)$ of patients with suspected lobular endocervical glandular hyperplasia showed a reduced lesion size after menopause. Lobular endocervical glandular hyperplasia has been reported to harbor estrogen receptor positive spindle cells in the stroma ${ }^{14}$; thus decreased serum levels of estrogen may be involved in the size reduction. There were two patients (Table 3 ) with suspected malignancy due to diffusion restriction on MRI indicated by a high signal intensity on diffusion weighted imaging and decreased intensity on the apparent diffusion coefficient map. However, these patients were diagnosed with lobular endocervical glandular hyperplasia and not cancer. Previous studies reported that no diffusion restriction was observed in malignant tumors associated with lobular endocervical glandular hyperplasia, ${ }^{15}{ }^{16}$ possibly due to the low cell density and low viscosity of intra-tumor mucins. In contrast, a study reported that diffusion restriction is important in the diagnosis of malignant tumors, ${ }^{17}$ and another reported that malignant tumors associated with lobular endocervical glandular hyperplasia exhibited diffusion restriction. ${ }^{15}$ In the present study, we could not deny the possibility of malignancy because of the presence of diffusion restriction. Further research is needed to evaluate the diagnostic potential of MRI in the detection of malignancy, especially those cases containing abundant mucin. It is not practical to follow women with suspected lobular endocervical glandular hyperplasia with periodic MRI. In addition, the positive predictive value of MRI alone for detecting malignancy is not high. Moreover, in the patient who later developed minimal deviation adenocarcinoma, cytology rather than MRI was effective in finding malignancy.

In conclusion, an increase in tumor size and worsening cytological findings are important parameters for detecting potentially malignant lobular endocervical glandular hyperplasia during follow-up. Furthermore, the frequency of malignant transformation of this lesion was small. Therefore, conservative management is an option for patients with this disease. Further research is needed to clarify the natural history, rate of malignant transformation, and useful clinical markers of malignant change.

Acknowledgements We thank Professor Fujinaga (Department of Radiology, Shinshu University School of Medicine, Japan), Professor Mikami (Department of Diagnostic Pathology, Kumamoto University Hospital, Japan), Dr Ishii (Department of Diagnostic Pathology, Okaya City Hospital, Japan), Dr Kashima (Department of Obstetrics and Gynecology, Shinonoi General Hospital, Japan), and all the members of the Department of Obstetrics and Gynecology, Department of Radiology, and Department of Laboratory Medicine in Shinshu University School of Medicine.

Contributors HK: data collection, data analysis, methodology, drafting, and editing. TM: project administration, data collection, review, and editing. HA and RA: data collection, data analysis, and review. AT: project administration, data collection, and review. A0: radiologist, radiological diagnosis, and review. SA: pathologist, pathological diagnosis, and review. TS: project administration, data analysis, review, and editing.

Funding This work was supported by Grant-in-Aid for Scientific Research (KAKENHI) from the Japan Society for the Promotion of Science (JSPS), grant No $15 \mathrm{~K} 10712$.

Competing interests None declared.

Patient consent for publication Not required.

Ethics approval The study was approved by the ethics committee of Shinshu University School of Medicine (No 4451), and the requirement for written informed consent was waived by the committee.

Provenance and peer review Not commissioned; externally peer reviewed.

Data availability statement Data are available upon reasonable request if the Ethics Committee of Shinshu University School of Medicine permits.

Open access This is an open access article distributed in accordance with the Creative Commons Attribution Non Commercial (CC BY-NC 4.0) license, which permits others to distribute, remix, adapt, build upon this work non-commercially, and license their derivative works on different terms, provided the original work is properly cited, an indication of whether changes were made, and the use is noncommercial. See: http://creativecommons.org/licenses/by-nc/4.0/.

ORCID iD

Tsutomu Miyamoto http://orcid.org/0000-0002-1513-9473

\section{REFERENCES}

1 Nucci MR, Clement PB, Young RH. Lobular endocervical glandular hyperplasia, not otherwise specified: a clinicopathologic analysis of thirteen cases of a distinctive pseudoneoplastic lesion and comparison with fourteen cases of adenoma malignum. Am J Surg Pathol 1999;23:886-91.

2 Wilbur DC, Colgan TJ, Ferenczy AS, et al. Glandular tumours and precursors. In: Kurman RJ, Carcangiu ML, Herrington CS, et al, eds. WHO classification of tumours of female reproductive organs. 4th edn. Lyon: International Agency for Research on Cancer, 2014: 183-94.

3 Takatsu A, Miyamoto T, Fuseya C, et al. Clonality analysis suggests that STK11 gene mutations are involved in progression of lobular endocervical glandular hyperplasia (LEGH) to minimal deviation adenocarcinoma (MDA). Virchows Arch 2013;462:645-51.

4 Mikami Y, Kiyokawa T, Hata S, et al. Gastrointestinal immunophenotype in adenocarcinomas of the uterine cervix 
and related glandular lesions: a possible link between lobular endocervical glandular hyperplasia/pyloric gland metaplasia and 'adenoma malignum'. Mod Pathol 2004;17:962-72.

5 Takatsu A, Shiozawa T, Miyamoto T, et al. Preoperative differential diagnosis of minimal deviation adenocarcinoma and lobular endocervical glandular hyperplasia of the uterine cervix: a multicenter study of clinicopathology and magnetic resonance imaging findings. Int J Gynecol Cancer 2011;21:1287-96.

6 Ando $\mathrm{H}$, Miyamoto $\mathrm{T}$, Kashima $\mathrm{H}$, et al. Usefulness of a management protocol for patients with cervical multicystic lesions: a retrospective analysis of 94 cases and the significance of Gnas mutation. J Obstet Gynaecol Res 2016;42:1588-98.

7 Ishii K, Kumagai T, Tozuka M, et al. A new diagnostic method for adenoma malignum and related lesions: latex agglutination test with a new monoclonal antibody, HIK1083. Clin Chim Acta 2001;312:231-3.

8 Ishii K, Katsuyama T, Ota H, et al. Cytologic and cytochemical features of adenoma malignum of the uterine cervix. Cancer Cytopathol 1999;87:245-53.

9 Kimura F, Kobayashi T, Kanai R, et al. Image quantification technology of the heterochromatin and euchromatin region for differential diagnosis in the lobular endocervical glandular hyperplasia. Diagn Cytopathol 2019;47:553-63.

10 Kobara H, Miyamoto T, Otsuki T, et al. Worsening cytology and lesion enlargement are useful indicators for malignant transformation of lobular endocervical glandular hyperplasia during follow-up: a case report. Gynecol Oncol Rep 2020;32.
11 Nishio S, Mikami Y, Tokunaga $\mathrm{H}$, et al. Analysis of gastric-type mucinous carcinoma of the uterine cervix - An aggressive tumor with a poor prognosis: A multi-institutional study. Gynecol Oncol 2019;153:13-19.

12 Ida K, Miyamoto T, Takatsu A, et al. Whole-exome sequencing of lobular endocervical glandular hyperplasia. Oncol Lett 2019;18:2592-7.

13 Sugihara T, Nakagawa S, Sasajima Y, et al. Case of minimal deviation adenocarcinoma: possible clinical link to lobular endocervical glandular hyperplasia as its origin. J Obstet Gynaecol Res 2015;41:483-7.

14 Mikami Y, Kiyokawa T, Moriya T, et al. Immunophenotypic alteration of the stromal component in minimal deviation adenocarcinoma ('adenoma malignum') and endocervical glandular hyperplasia: a study using oestrogen receptor and alpha-smooth muscle actin double immunostaining. Histopathology 2005;46:130-6.

15 Ohya A, Asaka S, Fujinaga Y, et al. Uterine cervical adenocarcinoma associated with lobular endocervical glandular hyperplasia: Radiologic-pathologic correlation. J Obstet Gynaecol Res 2018;44:312-22

16 Castán Senar A, Paño B, Saco A, et al. Magnetic resonance imaging of adenoma malignum of the uterine cervix with pathologic correlation: a case report. Radiol Case Rep 2016;11:323-7.

17 Koh D-M, Collins DJ. Diffusion-weighted MRI in the body: applications and challenges in oncology. AJR Am J Roentgenol 2007;188:1622-35. 